\title{
Highly Enantioenriched Homoenolate Reagents by Asymmetric $\gamma$-Deprotonation of Achiral 1-Silyl-Substituted 1-Alkenyl Carbamates
}

\author{
Jenny Reuber, Roland Fröhlich, and Dieter Hoppe* \\ Organisch-Chemisches Institut, Westfälische Wilhelms-Universität, Corrensstrasse 40, D-48149 Münster, \\ Germany, Fax: (+49)251-83-36531, dhoppe@uni-muenster.de
}

\section{Supporting Information}

\section{General Methods}

All organometallic reactions were performed under anhydrous conditions in an inert atmosphere of Argon in dried glassware. Solutions were transferred by means of syringes. Toluene and diethyl ether were dried with Na before use. Electrophiles were distilled prior to use. Analytical thin-layer chromatography was performed on Merck silica gel plates (Silica gel $60 \mathrm{~F}_{254}$ ). Visualization was accomplished with $\mathrm{UV}$ light and $\mathrm{KMnO}_{4}$ - or vanillin-solution. Column chromatography was carried out at 1.5 bar on silica gel 40-63 $\mu \mathrm{m}$ (Merck, Darmstadt). Only distilled solvents were used as eluents. ${ }^{1} \mathrm{H}$ NMR spectra and ${ }^{13} \mathrm{C}$ NMR spectra were recorded on a Bruker AM $300\left(300 \mathrm{MHz}\right.$ and $75.5 \mathrm{MHz}$ for ${ }^{1} \mathrm{H}$ and ${ }^{13} \mathrm{C}$ NMR, respectively). $\mathrm{CDCl}_{3}$ was used as solvent; chemical shifts are reported in ppm $(\delta) ;{ }^{1} \mathrm{H}$-shifts are related to TMS or $\mathrm{CDCl}_{3}$ and ${ }^{13} \mathrm{C}$-shifts to $\delta_{\mathrm{C}}=77.0 \mathrm{ppm}$; multiplicities are indicated by s (singlet), d (doublet), $\mathrm{t}$ (triplet), $\mathrm{q}$ (quartet), $\mathrm{m}$ (multiplet) and bs (broad singlet); * refers to signals which are interchangeable; brackets refer to signals which arise from frozen rotations of the amide bond. The numbering of the compounds can differ from the IUPAC name. Mass spectroscopy and elemental analysis were performed at the Institute of Organic Chemistry, University of Münster. IR absorption spectra were recorded using a Perkin-Elmer 298. The optical rotations were measured using a Perkin-Elmer polarimeter 241. Analytical capillary gas chromatography (GC) was performed using the following gas chromatograph, fitted with a flame detector: Hewlett Packard Series 5890 Series II, column HP5 and the following temperature programme: $50^{\circ} \mathrm{C}, 10^{\circ} \mathrm{C} / \mathrm{min}, 300^{\circ} \mathrm{C}, 10 \mathrm{~min}$. Chiral stationary phase HPLC was performed on a set with the following components: Waters 717 plus autosampler, Waters 600 Controller, Waters 996 Photodiode Array Detector and Waters In-Line Degasser (GROM Analytic + HPLC GmbH, Herrenberg, Germany).

\section{Detailed Reaction Procedures and Analytical Data of Representative Examples}

\section{Isomerization of Allyl Carbamates}

A solution of $N, N, N^{\prime}, N^{\prime}$-tetramethylethylenediamine (TMEDA, $36 \mathrm{mmol}, 4.184 \mathrm{~g}$,) in diethyl ether $(70 \mathrm{ml})$ was cooled to $-78^{\circ} \mathrm{C}$ and $n$ butyllithium $(1.6 \mathrm{M}$ in hexane, $36 \mathrm{mmol}, 22.5 \mathrm{ml})$ was added, followed after $10 \mathrm{~min}$ by a solution of the (E)-2-alkenyl $\mathrm{N}, \mathrm{N}$ diisopropylcarbamate $(\mathbf{1 5})(30 \mathrm{mmol})$ in diethyl ether $(10 \mathrm{ml})$. The solution was stirred for 1 hour at $-78^{\circ} \mathrm{C}$, then a solution of titanium tetraisopropoxide (39 mmol, $11.086 \mathrm{~g}$ ) in diethyl ether $(15 \mathrm{ml})$ was added dropwise. Stirring was continued for 1 hour before methanol $(25 \mathrm{ml})$ was added at $-78^{\circ} \mathrm{C}$ followed by $2 \mathrm{~N}$ aq. $\mathrm{HCl}(30 \mathrm{ml})$. After warming to r.t. and separation of the phases, the aqueous solution was extracted with diethyl ether $(3 \times 50 \mathrm{ml})$. The organic layers were combined and dried with $\mathrm{MgSO}_{4}$. After evaporation of the solvent in vacuo, the residue was purified by flash chromatography on silica gel (pentane/diethyl ether) which afforded the (Z)-1-alkenyl $N, N$-diisopropylcarbamate (14). 


\section{1 (Z)-1-Butenyl $N, N$-Diisopropylcarbamate (14a)}

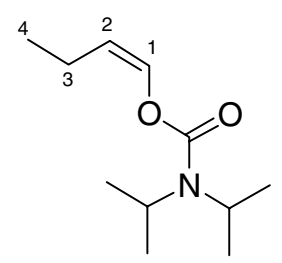

Isomerization of $(E)$-2-butenyl $N, N$-diisopropylcarbamate $(\mathbf{1 5 a})^{[1]}(30 \mathrm{mmol}, 5.979 \mathrm{~g})$ and purification (pentane/diethyl ether 20:1) yielded (Z)-1-butenyl $N, N$-diisopropylcarbamate (14a) as a colourless oil $(5.441 \mathrm{~g}, 91 \%)$.

$\mathrm{t}_{\mathrm{R}}=9.30 \mathrm{~min} ; \mathrm{R}_{\mathrm{f}}=0.40$ (pentane/diethyl ether $4: 1$ ).

${ }^{1} \mathrm{H}$ NMR (300 MHz, $\left.\mathrm{CDCl}_{3}\right): \delta / \mathrm{ppm}=1.01(\mathrm{t}, 3 \mathrm{H}, 4-\mathrm{H}) ; 1.26\left(\mathrm{~m}, 12 \mathrm{H}, \mathrm{CH}_{3}(\mathrm{Cb})\right.$ ); 2.17 (ddq, 2H, 3-H); 3.78 [4.14] (bs, 2H, CH(Cb)); 4.75 (dt, 1H, 2-H); 6.98 (dt, 1H, 1-H).

${ }^{3} J_{1,2}=6.4 \mathrm{~Hz} ;{ }^{4} J_{1,3}=1.6 \mathrm{~Hz} ;{ }^{3} J_{2,3}=7.3 \mathrm{~Hz} ;{ }^{3} J_{3,4}=7.5 \mathrm{~Hz}$.

${ }^{13} \mathrm{C} \mathrm{NMR}\left(75 \mathrm{MHz}, \mathrm{CDCl}_{3}\right): \delta / \mathrm{ppm}=14.1(\mathrm{C}-4) ; 18.3(\mathrm{C}-3) ; 20.4[21.1]\left(\mathrm{CH}_{3}(\mathrm{Cb})\right)$;

45.6 [46.8] (CH(Cb)); $112.7(\mathrm{C}-2) ; 134.4(\mathrm{C}-1) ; 153.5(\mathrm{OCON})$.

IR (neat): $\tilde{v} / \mathrm{cm}^{-1}=2968,2931,2871,1699,1470,1438,1314,1291,1244,1148,1139$, 1090, 1072, 1049.

$\operatorname{MS}(\mathrm{ESI})(\mathrm{m} / \mathrm{z}):[\mathrm{M}+\mathrm{Na}]^{+}=222.3$.

Elemental analysis: calcd.: C 66.29 H $10.62 \quad$ N 7.03

$\mathrm{C}_{11} \mathrm{H}_{21} \mathrm{NO}_{2} \quad$ found: $\mathrm{C} 66.14 \quad \mathrm{H} 10.91 \quad \mathrm{~N} 7.00$.

\section{$1.2(Z)-3-P h e n y l-1-p r o p e n y l ~ N, N$-Diisopropylcarbamate (14b)}

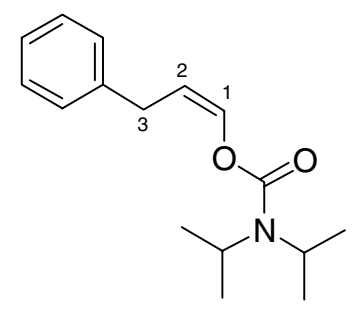

Isomerization of (E)-3-phenyl-2-propenyl $N, N$-diisopropylcarbamate $(\mathbf{1 5 b})^{[2]}$ (30 mmol, $7.841 \mathrm{~g}$ ) and purification (pentane/diethyl ether $6: 1 \rightarrow 4: 1$ ) afforded $(Z)$-3-phenyl-1-propenyl $N, N$-diisopropylcarbamate (14b) as a yellow oil $(6.273 \mathrm{~g}, 80 \%)$.

$\mathrm{t}_{\mathrm{R}}=15.69 \mathrm{~min} ; \mathrm{R}_{\mathrm{f}}=0.25$ (pentane/diethyl ether $=6: 1$ ).

${ }^{1} \mathrm{H}$ NMR $\left(300 \mathrm{MHz}, \mathrm{CDCl}_{3}\right): \delta / \mathrm{ppm}=1.26\left(\mathrm{~m}, 12 \mathrm{H}, \mathrm{CH}_{3}(\mathrm{Cb})\right) ; 3.52(\mathrm{~d}, 2 \mathrm{H}, 3-\mathrm{H}) ; 3.84$ [4.07] (bs, 2H, $\mathrm{CH}(\mathrm{Cb})) ; 4.94(\mathrm{dt}, 1 \mathrm{H}, 2-\mathrm{H}) ; 7.14-7.28(\mathrm{~m}, 6 \mathrm{H}$, aryl-H/1-H).

${ }^{3} J_{1,2}=7.5 \mathrm{~Hz} ;{ }^{3} J_{2,3}=6.4 \mathrm{~Hz}$.

\footnotetext{
${ }^{1}$ Hoppe, D.; Hanko, R.; Brönneke, A.; Lichtenberg, F.; van Hülsen, E. Chem. Ber. 1985, 118, 2822.

${ }^{2}$ Hoppe, D.; Behrens, K.; Fröhlich, R.; Meyer, O. Eur. J. Org. Chem. 1998, 2397.
} 
${ }^{13} \mathrm{C} \mathrm{NMR}\left(75 \mathrm{MHz}, \mathrm{CDCl}_{3}\right): \delta / \mathrm{ppm}=20.5$ [21.4] $\left(\mathrm{CH}_{3}(\mathrm{Cb})\right) ; 31.2(\mathrm{C}-3) ; 45.7$ [46.6]

$(\mathrm{CH}(\mathrm{Cb})) ; 109.2$ (C-2); 126.0/128.2/128.3 (C-aryl); 135.7 (C-1); 140.5 (Cq-aryl); 152.8 (OCON).

IR (neat): $\tilde{v} / \mathrm{cm}^{-1}=2972,2933,2873,1709,1470,1437,1373,1313,1286,1244,1216$, 1160, 1134, 1091, 1072, 1049, 750, 699.

$\operatorname{MS}(\mathrm{ESI})(\mathrm{m} / \mathrm{z}):[\mathrm{M}+\mathrm{Na}]^{+}=284.5$.

Elemental analysis: calcd.: C 73.53 H 8.87 $\quad$ N 5.36

$\mathrm{C}_{16} \mathrm{H}_{23} \mathrm{NO}_{2} \quad$ found: $\mathrm{C} 73.55 \quad \mathrm{H} 8.90 \quad \mathrm{~N}$ 5.25.

\section{Deprotonation of 1-Alkenyl carbamates $14^{[3]}$ and Addition of Trimethylsilyl Chloride}

To a solution of TMEDA (22 mmol, $2.557 \mathrm{~g})$ in diethyl ether $(60 \mathrm{ml}) n$ butyllithium $(1.6 \mathrm{M}$ in hexane, $22 \mathrm{mmol}, 13.8 \mathrm{ml}$ ) was added at $-78^{\circ} \mathrm{C}$, followed after $10 \mathrm{~min}$ by a solution of the (Z)-1-alkenyl $N, N$-diisopropylcarbamate (14) $(20 \mathrm{mmol})$ in diethyl ether $(10 \mathrm{ml})$. The solution was stirred for 1 hour at $-78^{\circ} \mathrm{C}$. A solution of trimethylsilyl chloride $(24 \mathrm{mmol}, 2.609 \mathrm{~g})$ in diethyl ether $(8 \mathrm{ml})$ was added slowly within $15 \mathrm{~min}$. Stirring was continued for 1 hour before the reaction was stopped by addition of sat. ammonium chloride solution $(25 \mathrm{ml})$ at $-78^{\circ} \mathrm{C}$. After warming to room temperature and separation of the phases, the aqueous solution was extracted with diethyl ether $(3 \times 50 \mathrm{ml})$ and dried with $\mathrm{MgSO}_{4}$. After evaporation of the solvent in vacuo, the residue was purified by flash chromatography on silica gel (pentane/diethyl ether 20:1) which afforded the (E)-1-trimethylsilyl-1-alkenyl $N, N$-diisopropylcarbamate (1).

\section{1 (E)-1-Trimethylsilyl-1-butenyl $N, N$-Diisopropylcarbamate (1a)}

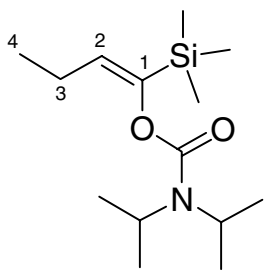

Deprotonation of (Z)-1-butenyl $N, N$-diisopropylcarbamate (14a) $(20 \mathrm{mmol}, 3.986 \mathrm{~g})$ and substitution with trimethylsilyl chloride yielded (E)-1-trimethylsilyl-1-butenyl $\mathrm{N}, \mathrm{N}$ diisopropylcarbamate (1a) as a colourless oil (3.746 g, 69\%).

$t_{\mathrm{R}}=10.47 \mathrm{~min} ; \mathrm{R}_{\mathrm{f}}=0.40$ (pentane/diethyl ether $=4: 1$ ).

${ }^{1} \mathrm{H}$ NMR $\left(300 \mathrm{MHz}, \mathrm{CDCl}_{3}\right): \delta / \mathrm{ppm}=0.10\left(\mathrm{~s}, 9 \mathrm{H}, \mathrm{CH}_{3} \mathrm{Si}\right) ; 0.94(\mathrm{t}, 3 \mathrm{H}, 4-\mathrm{H}) ; 1.21(\mathrm{~m}, 12 \mathrm{H}$, $\left.\mathrm{CH}_{3}(\mathrm{Cb})\right) ; 2.07$ (dq, 2H, 3-H); 3.78 [4.04] (bs, 2H, CH(Cb)); $5.32(\mathrm{t}, 1 \mathrm{H}, 2-\mathrm{H})$.

${ }^{3} J_{2,3}=7.0 \mathrm{~Hz} ;{ }^{3} J_{3,4}=7.6 \mathrm{~Hz}$.

${ }^{13} \mathrm{C}$ NMR $\left(75 \mathrm{MHz}, \mathrm{CDCl}_{3}\right): \delta / \mathrm{ppm}=-0.8\left(\mathrm{CH}_{3} \mathrm{Si}\right) ; 13.6(\mathrm{C}-4) ; 19.3(\mathrm{C}-3) ; 20.0$ [21.5] $\left(\mathrm{CH}_{3}(\mathrm{Cb})\right) ; 45.7$ [46.7] $(\mathrm{CH}(\mathrm{Cb})) ; 131.5(\mathrm{C}-2) ; 154.1(\mathrm{C}-1) * ; 154.3(\mathrm{OCON}) *$.

IR (neat): $\tilde{v} / \mathrm{cm}^{-1}=2968,2935,2899,1701,1435,1334,1246,1137,1068,841,767$.

$\operatorname{ESI-MS~}(\mathrm{m} / \mathrm{z}):[\mathrm{M}+\mathrm{H}]^{+}=272.2 ;[\mathrm{M}+\mathrm{Na}]^{+}=294.2 ;[\mathrm{M}+\mathrm{K}]^{+}=310.2$.

\footnotetext{
${ }^{3}$ Snieckus, V.; Sengupta, S. J. Org. Chem. 1990, 55, 5680.
} 
HRMS:

calcd.: $[\mathrm{M}+\mathrm{H}]^{+}=272.2046 ;[\mathrm{M}+\mathrm{Na}]^{+}=294.1865$

$\mathrm{C}_{14} \mathrm{H}_{29} \mathrm{NO}_{2} \mathrm{Si} \quad$ found: $[\mathrm{M}+\mathrm{H}]^{+}=272.2026 ;[\mathrm{M}+\mathrm{Na}]^{+}=294.1906$.

\section{2 (E)-3-Phenyl-1-trimethylsilyl-1-propenyl $N, N$-Diisopropylcarbamate (1b)}<smiles>CC(C)N(C(=O)O/C(=C\Cc1ccccc1)[Si](C)(C)C)C(C)C</smiles>

Deprotonation of (Z)-3-phenyl-1-propenyl $N, N$-diisopropylcarbamate (14b) (20 mmol, $5.230 \mathrm{~g})$ and substitution with trimethylsilyl chloride yielded (E)-3-Phenyl-1-trimethylsilyl-1propenyl $N, N$-diisopropylcarbamate (1b) as a colourless oil $(5.537 \mathrm{~g}, 83 \%)$.

$\mathrm{t}_{\mathrm{R}}=15.95 \mathrm{~min} ; \mathrm{R}_{\mathrm{f}}=0.37$ (pentane/diethyl ether $=6: 1$ ).

${ }^{1} \mathrm{H}$ NMR (300 MHz, $\left.\mathrm{CDCl}_{3}\right): \delta / \mathrm{ppm}=0.00\left(\mathrm{~s}, 9 \mathrm{H}, \mathrm{CH}_{3} \mathrm{Si}\right) ; 0.90\left(\mathrm{~d}, 12 \mathrm{H}, \mathrm{CH}_{3}(\mathrm{Cb})\right) ; 3.30$ $(\mathrm{d}, 2 \mathrm{H}, 3-\mathrm{H}) ; 3.68$ [3.88] (bs, 2H, $\mathrm{CH}(\mathrm{Cb})) ; 5.37$ (t, 1H, 2-H); 7.01-7.13 (m, 5H, aryl-H).

${ }^{3} J_{C b}=6.9 \mathrm{~Hz} ;{ }^{3} J_{2,3}=7.0 \mathrm{~Hz}$.

${ }^{13} \mathrm{C}$ NMR (75 MHz, $\left.\mathrm{CDCl}_{3}\right): \delta / \mathrm{ppm}=-0.8\left(\mathrm{CH}_{3} \mathrm{Si}\right) ; 20.5$ [21.5] $\left(\mathrm{CH}_{3}(\mathrm{Cb})\right) ; 32.2(\mathrm{C}-3) ; 45.8$ [46.4] $(\mathrm{CH}(\mathrm{Cb})) ; 125.9$ (C-2); 127.9/128.4/128.5 (CH-aryl); 141.2 (Cq-aryl); 154.0 $(\mathrm{OCON})^{*} ; 155.6(\mathrm{C}-1)^{*}$.

IR (neat): $\tilde{v} / \mathrm{cm}^{-1}=3025,2972,2904,1699,1437,1368,1328,1293,1248,1213,1161$, 1135, 1095, 1074, 1150, 842, 764, 748, 703.

MS (ESI) $(\mathrm{m} / \mathrm{z}): \quad[\mathrm{M}+\mathrm{H}]^{+}=334.7 ;[\mathrm{M}+\mathrm{Na}]^{+}=356.6$.

Elemental analysis: $\quad$ calcd.: C $68.42 \quad$ H $9.37 \quad$ N 4.20

$\mathrm{C}_{19} \mathrm{H}_{31} \mathrm{NO}_{2} \mathrm{Si} \quad$ found: $\mathrm{C} 68.39 \quad \mathrm{H} 9.25 \quad \mathrm{~N} 4.15$.

\section{Lithiation and Electrophilic Substitution of 1a}

\section{$3.1(+)-(1 E, 3 S)-1,3-B i s(t r i m e t h y l s i l y l)-1-b u t e n y l ~ N, N$-Diisopropylcarbamate (4a)}

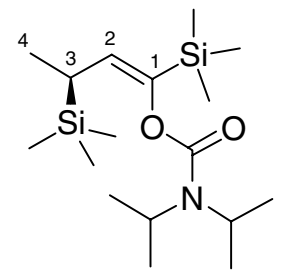

A solution of (-)-sparteine $(1.5 \mathrm{mmol}, 352 \mathrm{mg})$ in toluene $(2 \mathrm{ml})$ was cooled to $-78^{\circ} \mathrm{C}$. nbutyllithium (1.6 M in hexane, $1.5 \mathrm{mmol}, 0.94 \mathrm{ml}$ ) was added followed by a solution of (E)-1-trimethylsilyl-1-butenyl $\mathrm{N}, \mathrm{N}$-diisopropylcarbamate (1a) $(0.5 \mathrm{mmol}, 136 \mathrm{mg})$ in toluene $(1.0 \mathrm{ml})$ after $10 \mathrm{~min}$ and the clear yellow solution was stirred for 12 hours. A solution of 
trimethylsilyl chloride $(4.5 \mathrm{mmol}, 489 \mathrm{mg})$ in toluene $(1.0 \mathrm{ml})$ was added slowly within $10 \mathrm{~min}$. The solution was stirred additional $2 \mathrm{~h}$ and then the reaction was quenched by addition of a mixture of glacial acid $(0.05 \mathrm{ml})$ and methanol $(0.3 \mathrm{ml})$ at $-78^{\circ} \mathrm{C}$. After warming to r.t. and dilution of the organic phase with diethyl ether $(10 \mathrm{ml})$ it was dried with $\mathrm{MgSO}_{4}$ and the solvent was removed in vacuo. Purification by column chromatography (pentane/diethyl ether 20:1) furnished (1E,3S)-1,3-bis(trimethylsilyl)-1-butenyl $\mathrm{N}, \mathrm{N}$-diisopropylcarbamate (4a) $(146 \mathrm{mg}, 85 \%)$.

$t_{R}=13.16 \min ; R_{f}=0.60$ (pentane/diethyl ether $=6: 1$ ).

$[\alpha]_{D}^{20}=+32\left(\mathrm{c}=1.31\right.$ in $\mathrm{CHCl}_{3} ; \geq 95 \%$ ee $)$.

${ }^{1} \mathrm{H}$ NMR shift-experiment: $39 \mathrm{~mol} \% \mathrm{Eu}(\mathrm{hfc})_{3}$ in $\mathrm{CDCl}_{3}, \Delta \delta\left(\mathrm{CH}_{3} \mathrm{Si}\right)=0.01 \mathrm{ppm}$ (major enantiomer: $\delta=0.17 \mathrm{ppm}$, minor enantiomer: $\delta=0.18 \mathrm{ppm})$.

${ }^{1} \mathrm{H}$ NMR $\left(300 \mathrm{MHz}, \mathrm{CDCl}_{3}\right): \delta / \mathrm{ppm}=-0.05$ (s, 9H, $\left.\mathrm{CH}_{3} \mathrm{Si}\right) ; 0.12\left(\mathrm{~s}, 9 \mathrm{H}, \mathrm{CH}_{3} \mathrm{Si}\right) ; 1.03$ (d, 3H, 4-H); 1.23 (d, 12H, $\left.\mathrm{CH}_{3}(\mathrm{Cb})\right) ; 2.07$ (dq, 1H, 3-H); 3.92 (bs, 2H, $\left.\mathrm{CH}(C b)\right) ; 5.10$ (d, 1H, 2-H);

${ }^{3} J_{C b}=6.6 \mathrm{~Hz} ;{ }^{3} J_{2,3}=10.7 \mathrm{~Hz} ;{ }^{3} J_{3,4}=7.2 \mathrm{~Hz}$.

${ }^{13} \mathrm{C} \mathrm{NMR}\left(75 \mathrm{MHz}, \mathrm{CDCl}_{3}\right): \delta / \mathrm{ppm}=0.4\left(\mathrm{CH}_{3} \mathrm{Si}\right) ; 14.6(\mathrm{C}-4) ; 21.0(\mathrm{C}-3) ; 21.4$ [20.6]

$\left(\mathrm{CH}_{3}(\mathrm{Cb})\right) ; 46.0(\mathrm{CH}(\mathrm{Cb})) ; 132.7(\mathrm{C}-2) ; 151.6(\mathrm{C}-1) ; 154.1$ (OCON).

IR (neat): $\tilde{v} / \mathrm{cm}^{-1}=2960,2900,2870,1702,1433,1333,1291,1247,1131,1070,1048$, $839,767,751$.

$\operatorname{MS}(\mathrm{ESI})(\mathrm{m} / \mathrm{z}): \quad[\mathrm{M}+\mathrm{H}]^{+}=344.3 ;[\mathrm{M}+\mathrm{Na}]^{+}=366.2 ;[\mathrm{M}+\mathrm{K}]^{+}=382.2$.

Elemantal analysis: calcd.: C 59.42 $\quad$ H $10.85 \quad$ N 4.08

$\begin{array}{llll}\mathrm{C}_{17} \mathrm{H}_{37} \mathrm{NO}_{2} \mathrm{Si}_{2} & \text { found: } \mathrm{C} 59.20 & \mathrm{H} 11.03 & \mathrm{~N} 3.78 \text {. }\end{array}$

\section{2 (-)-(1E,3S,4R)-4-Hydroxy-3,5-dimethyl-1-trimethylsilyl-1-hexenyl $N, N$ - Diisopropylcarbamate (anti-6b)}

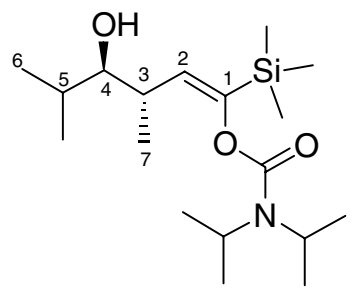

(E)-1-Trimethylsilyl-1-butenyl $N, N$-diisopropylcarbamate (1a) $(0.5 \mathrm{mmol}, 136 \mathrm{mg})$ was lithiated as described above (3.1). After titanation with a solution of ClTi( $\left.\mathrm{NEt}_{2}\right)_{3}(1.75 \mathrm{mmol}$, $525 \mathrm{mg}$ ) in toluene $(1 \mathrm{ml})$ for $6 \mathrm{~h}$, the red-brown intermediate was trapped with a solution of 2-methylpropanal $(3.5 \mathrm{mmol}, 252 \mathrm{mg})$ in toluene $(0.5 \mathrm{ml})$. The solution was then allowed to warm to $-25^{\circ} \mathrm{C}$ and stirred additional $2 \mathrm{~h}$. During this time period the solution became orange. The reaction was stopped by the addition of a mixture of methanol $(0.5 \mathrm{ml})$ and glacial acid $(0.1 \mathrm{ml})$ at $-78^{\circ} \mathrm{C}$. The mixture was warmed to r.t., poured into $2 \mathrm{~N}$ aq. $\mathrm{HCl}(10 \mathrm{ml})$, extracted with diethyl ether $(3 \times 10 \mathrm{ml})$, and dried with $\mathrm{MgSO}_{4}$. Evaporation of the solvent and chromatography of the crude product (cyclohexane/ethyl acetate 10:1) afforded (-)-(1E,3S,4R)-4-hydroxy-3,5-dimethyl-1-trimethylsilyl-1-hexenyl $N, N$-diisopropylcarbamate (anti-6b) as a colourless oil (120 mg, 70\%).

$t_{R}=14.90 \min ; R_{f}=0.13$ (pentane/diethyl ether 5:1). 
$[\alpha]_{D}^{20}=-27\left(\mathrm{c}=0.97\right.$ in $\mathrm{CHCl}_{3} ; \geq 95 \%$ ee $)$.

${ }^{1} \mathrm{H}$ NMR shift-experiment: $27 \mathrm{~mol} \% \mathrm{Eu}(\mathrm{hfc})_{3}$ in $\mathrm{CDCl}_{3}, \Delta \delta\left(\mathrm{CH}_{3} \mathrm{Si}\right)=0.04 \mathrm{ppm}$ (major enantiomer: $\delta=0.33 \mathrm{ppm}$, minor enantiomer: $\delta=0.29 \mathrm{ppm})$.

${ }^{1} \mathrm{H}$ NMR $\left(300 \mathrm{MHz}, \mathrm{CDCl}_{3}\right): \delta / \mathrm{ppm}=0.12\left(\mathrm{~s}, 9 \mathrm{H}, \mathrm{CH}_{3} \mathrm{Si}\right) ; 0.81(\mathrm{~d}, 3 \mathrm{H}, 7-\mathrm{H}) ; 0.92 / 0.98$ (d, $6 \mathrm{H}, 6-\mathrm{H}) ; 1.16-1.23\left(\mathrm{~m}, 12 \mathrm{H}, \mathrm{CH}_{3}(\mathrm{Cb})\right)$; 1.67-1.77 (m, 1H, 5-H); 2.61-2.72 (m, 1H, 3-H); 2.84-2.85 (m, 1H, OH); 3.07-3.12 (m, 1H, 4-H); $3.93(\mathrm{~m}, 2 \mathrm{H}, \mathrm{CH}(\mathrm{Cb})) ; 5.37(\mathrm{~d}, 1 \mathrm{H}, 2-\mathrm{H})$. ${ }^{3} J_{2,3}=6.7 \mathrm{~Hz} ;{ }^{3} J_{6,5}=6.6 \mathrm{~Hz}$.

${ }^{13} \mathrm{C}$ NMR $\left(75 \mathrm{MHz}, \mathrm{CDCl}_{3}\right): \delta / \mathrm{ppm}=-0.8\left(\mathrm{CH}_{3} \mathrm{Si}\right) ; 15.3 / 17.9 / 20.8(\mathrm{C}-6 / \mathrm{C}-7) ; 21.0$ [21.8]

$\left(\mathrm{CH}_{3}(\mathrm{Cb})\right) ; 30.6(\mathrm{C}-5)^{*} ; 34.9(\mathrm{C}-3)^{*} ; 46.5$ [47.2] $(\mathrm{CH}(\mathrm{Cb})) ; 79.8(\mathrm{C}-4) ; 134.7(\mathrm{C}-2) ; 155.0$ $(\mathrm{OCON})^{*} ; 156.1(\mathrm{C}-1)^{*}$.

IR (neat): $\tilde{v} / \mathrm{cm}^{-1}=3444,2969,2935,2903,2875,1683,1436,1335,1305,1248,1214$, $1153,1051,1002,843,764$.

$\operatorname{MS}(\mathrm{ESI})(\mathrm{m} / \mathrm{z}): \quad[\mathrm{M}+\mathrm{H}]^{+}=344 ;[\mathrm{M}+\mathrm{Na}]^{+}=366 ;[\mathrm{M}+\mathrm{K}]^{+}=368$.

Elemantal analysis: calcd.: C $62.92 \quad$ H $10.85 \quad$ N 4.08

$\begin{array}{llll}\mathrm{C}_{18} \mathrm{H}_{37} \mathrm{NO}_{3} \mathrm{Si} & \mathrm{H} 10.88 & \mathrm{~N} \\ & \text { found: } \mathrm{C} 62.80\end{array}$

\section{Lithiation and Electrophilic Substitution of $1 \mathrm{~b}$}

4.1 (+)-(1E,3S)-3-Methoxycarbonyl-3-phenyl-1-trimethylsilyl-1-propenyl $N, N$ Diisopropylcarbamate (ent-9d)

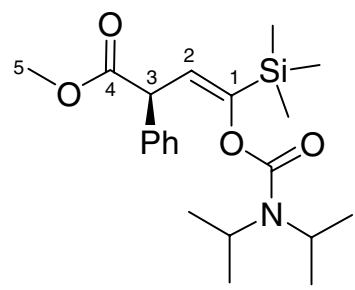

To a stirred solution of (-)-sparteine $(1.5 \mathrm{mmol}, 352 \mathrm{mg})$ in toluene $(2 \mathrm{ml})$ was added $n$ butyllithium $(1.6 \mathrm{M}$ in hexane, $1.5 \mathrm{mmol}, 0.94 \mathrm{ml})$ at $-78^{\circ} \mathrm{C}$. After $10 \mathrm{~min}(E)$-3-phenyl-1trimethylsilyl-1-propenyl $N, N$-diisopropylcarbamate (1b) $(0.5 \mathrm{mmol}, 167 \mathrm{mg})$ was added dropwise and the clear yellow solution was stirred for 1 hour. A solution of methyl chloroformate $(1.5 \mathrm{mmol}, 142 \mathrm{mg})$ in toluene $(0.5 \mathrm{ml})$ was added dropwise over $10 \mathrm{~min}$. The mixture became colourless within $2 \mathrm{~h}$ and was quenched with a mixture of glacial acid $(0.05 \mathrm{ml})$ and methanol $(0.3 \mathrm{ml})$. After warming to r.t. and dilution of the organic phase with diethyl ether it was dried with $\mathrm{MgSO}_{4}$ and the solvent was removed in vacuo. Purification by column chromatography (pentane/diethyl ether 6:1) furnished (+)-(1E,3S)-3methoxycarbonyl-3-phenyl-1-trimethylsilyl-1-propenyl $N, N$-diisopropylcarbamate (ent-9d) (141 mg, 72\%).

$t_{R}=17.57 \mathrm{~min} ; \mathrm{R}_{\mathrm{f}}=0.27$ (pentane/diethyl ether $=4: 1$ ).

$[\alpha]_{D}^{20}=+76\left(\mathrm{c}=0.77\right.$ in $\mathrm{CHCl}_{3} ; \geq 95 \%$ ee $)$.

${ }^{1} \mathrm{H}$ NMR shift-experiment: $20 \mathrm{~mol} \% \mathrm{Eu}(\mathrm{hfc})_{3}$ in $\mathrm{CDCl}_{3}, \Delta \delta\left(\mathrm{CH}_{3} \mathrm{Si}\right)=0.01 \mathrm{ppm}$ (major enantiomer: $\delta=0.23 \mathrm{ppm}$, minor enantiomer: $\delta=0.24 \mathrm{ppm}$ ); $\Delta \delta(5-\mathrm{H})=0.02 \mathrm{ppm}$ (major enantiomer: $\delta=3.82 \mathrm{ppm}$, minor enantiomer: $\delta=3.84 \mathrm{ppm}$ ). 
${ }^{1} \mathrm{H}$ NMR (300 MHz, $\left.\mathrm{CDCl}_{3}\right): \delta / \mathrm{ppm}=-0.01\left(\mathrm{~s}, 9 \mathrm{H}, \mathrm{CH}_{3} \mathrm{Si}\right) ; 1.01-1.05\left(\mathrm{~m}, 12 \mathrm{H}, \mathrm{CH}_{3}(\mathrm{Cb})\right.$ ); 3.50 (s, 3H, 5-H); 3.70 (bs, 2H, CH(Cb)); 4.58 (d, 1H, 3-H); $5.66(\mathrm{~d}, 1 \mathrm{H}, 2-\mathrm{H}) ; 7.05-7.13$ (m, $5 \mathrm{H}$, aryl-H).

${ }^{3} J_{2,3}=8.8 \mathrm{~Hz}$.

${ }^{13} \mathrm{C}$ NMR $\left(75 \mathrm{MHz}, \mathrm{CDCl}_{3}\right): \delta / \mathrm{ppm}=-0.6\left(\mathrm{CH}_{3} \mathrm{Si}\right) ; 20.4[21.4]\left(\mathrm{CH}_{3}(\mathrm{Cb})\right) ; 46.3(\mathrm{CH}(\mathrm{Cb}))$; 48.2 (C-3); 52.1 (C-5); 125.0 (C-2); 127.1/127.8/128.6 (CH-aryl); 138.6 (Cq-aryl); 153.2 $(\mathrm{OCON})^{*} ; 157.1(\mathrm{C}-1)^{*} ; 172.7(\mathrm{C}-4)$.

IR (neat): $\tilde{v} / \mathrm{cm}^{-1}=2971,2899,1744,1698,1471,1457,1438,1370,1245,1214,1193$, 1161, 1134, 1073, 1060, 1044, 839, 773, 730, 701.

MS (ESI) (m/z): $[\mathrm{M}+\mathrm{Na}]^{+}=414.3$.

Elemental analysis: calcd.: C $64.41 \quad$ H $8.49 \quad$ N 3.58

$\begin{array}{llll}\mathrm{C}_{21} \mathrm{H}_{33} \mathrm{NO}_{4} \mathrm{Si} & \text { found: } \mathrm{C} 64.54 & \mathrm{H} \text { 8 } 68 & \text { N 3.49. }\end{array}$

\section{$4.2(-)-(1 E, 3 R, 4 R)-4-H y d r o x y-5-m e t h y l-3-p h e n y l-1-t r i m e t h y l s i l y l-1-h e x e n y l ~ N, N$ - Diisopropylcarbamate (anti-11b)}

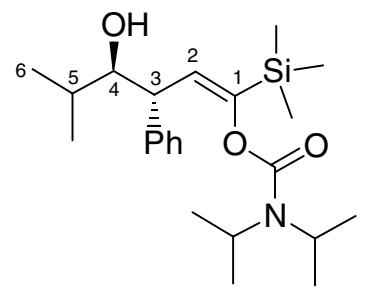

(E)-3-Phenyl-1-trimethylsilyl-1-propenyl $N, N$-diisopropylcarbamate (1b) $(0.5 \mathrm{mmol}, 167 \mathrm{mg})$ was lithiated as described above (4.1). After titanation with a solution of $\operatorname{ClTi}\left(\mathrm{NEt}_{2}\right)_{3}$ $(0.75 \mathrm{mmol}, 225 \mathrm{mg})$ in toluene $(0.5 \mathrm{ml})$ for $30 \mathrm{~min}$, the red-brown coloured intermediate was trapped with a solution of 2-methylpropanal $(2.0 \mathrm{mmol}, 144 \mathrm{mg})$ in toluene $(0.5 \mathrm{ml})$. Within $2 \mathrm{~h}$ the solution became orange. The reaction was stopped by the addition of methanol $(0.5 \mathrm{ml})$ and glacial acid $(0.1 \mathrm{ml})$ at $-78^{\circ} \mathrm{C}$. The mixture was warmed to r.t. and was poured into $2 \mathrm{~N}$ aq. $\mathrm{HCl}(10 \mathrm{ml})$. The aqueous phase was extracted with diethyl ether $(3 \times 10 \mathrm{ml})$ and the ethereal extracts were dried with $\mathrm{MgSO}_{4}$ and the solvent was removed in vacuo. Purification of the crude product by chromatography (cyclohexane/ethyl acetate 10:1) afforded (-)-(1E,3R,4R)-4-hydroxy-5-methyl-3-phenyl-1-trimethylsilyl-1-hexenyl $N, N$ diisopropylcarbamate (anti-11b) as a colourless oil (193 mg, 95\%).

$\mathrm{t}_{\mathrm{R}}=18.12 \mathrm{~min} ; \mathrm{R}_{\mathrm{f}}=0.45($ ether/pentane $=1: 1)$.

$[\alpha]_{D}^{20}=-162\left(\mathrm{c}=0.78\right.$ in $\left.\mathrm{CHCl}_{3} ; 94 \% e e\right)$.

Chiral HPLC (chiral stationary phase: CHIRA-Grom-2, GROM Analytic + HPLC GmbH, Herrenberg, Germany): isopropanol $/$ hexane $=1: 1000 ; 0.3 \mathrm{ml} / \mathrm{min} ;$ major enantiomer: $\mathrm{t}_{\mathrm{R} 1}=$ 10.13 min and minor enantiomer: $t_{\mathrm{R} 2}=9.01 \mathrm{~min}$.

${ }^{1} \mathrm{H}$ NMR $\left(300 \mathrm{MHz}, \mathrm{CDCl}_{3}\right): \delta / \mathrm{ppm}=0.00\left(\mathrm{~s}, 9 \mathrm{H}, \mathrm{CH}_{3} \mathrm{Si}\right) ; 0.69 / 0.78(\mathrm{~d}, 6 \mathrm{H}, 6-\mathrm{H}) ; 1.11-1.20$ $\left(\mathrm{m}, 12 \mathrm{H}, \mathrm{CH}_{3}(\mathrm{Cb})\right) ; 1.31\left(\mathrm{~m}_{\mathrm{c}}, 1 \mathrm{H}, 5-\mathrm{H}\right) ; 3.17(\mathrm{~m}, 1 \mathrm{H}, \mathrm{OH}) ; 3.47-3.53(\mathrm{~m}, 1 \mathrm{H}, 3-\mathrm{H}) ; 3.68$ (dd, $1 \mathrm{H}, 4-\mathrm{H}) ; 3.78$ [3.94] (m, 2H, $\mathrm{CH}(\mathrm{Cb})) ; 5.69(\mathrm{~d}, 1 \mathrm{H}, 2-\mathrm{H}) ; 7.04-7.19(\mathrm{~m}, 5 \mathrm{H}$, aryl-H).

${ }^{3} J_{2,3}=10.5 \mathrm{~Hz} ;{ }^{3} J_{3,4}=9.6 \mathrm{~Hz} ;{ }^{3} J_{4,5}=9.6 \mathrm{~Hz} ;{ }^{3} J_{5,6}=6.7 \mathrm{~Hz}$.

${ }^{13} \mathrm{C}$ NMR $\left(75 \mathrm{MHz}, \mathrm{CDCl}_{3}\right): \delta / \mathrm{ppm}=-1.5\left(\mathrm{CH}_{3} \mathrm{Si}\right) ; 14.7 / 20.4(\mathrm{C}-6) ; 20.3[21.6]\left(\mathrm{CH}_{3}(\mathrm{Cb})\right)$; 29.7 (C-5); 46.3 (C-3); 45.9[46.7] $(\mathrm{CH}(\mathrm{Cb})) ; 78.8$ (C-4); 126.5/127.9/128.7 (CH-aryl); 131.8 (C-2); 141.7 (Cq-aryl); $154.4(\mathrm{OCON})^{*} ; 156.2(\mathrm{C}-1)^{*}$. 
IR (neat): $\tilde{v} / \mathrm{cm}^{-1}=3273,2826,1620,1385,1324,1272,1236,1201,1170,1102,1014$, 969, 813, 728, 675, 643.

MS (ESI) (m/z): $[\mathrm{M}+\mathrm{H}]^{+}=406.4 ;[\mathrm{M}+\mathrm{Na}]^{+}=428.4$.

$\begin{array}{llll}\text { Elemental analysis: } & \text { calcd.: } \mathrm{C} 68.10 & \mathrm{H} 9.69 & \mathrm{~N} 3.45 \\ \mathrm{C}_{23} \mathrm{H}_{39} \mathrm{NO}_{3} \mathrm{Si} & \text { found: } \mathrm{C} 67.81 & \mathrm{H} 9.40 & \text { N } 3.33 .\end{array}$

\section{$5{ }^{1}$ H NMR spectra for important compounds}

\section{$5.1(+)-(1 E, 3 S)-1,3-B i s($ trimethylsilyl)-1-butenyl $N, N$-Diisopropylcarbamate (4a)}

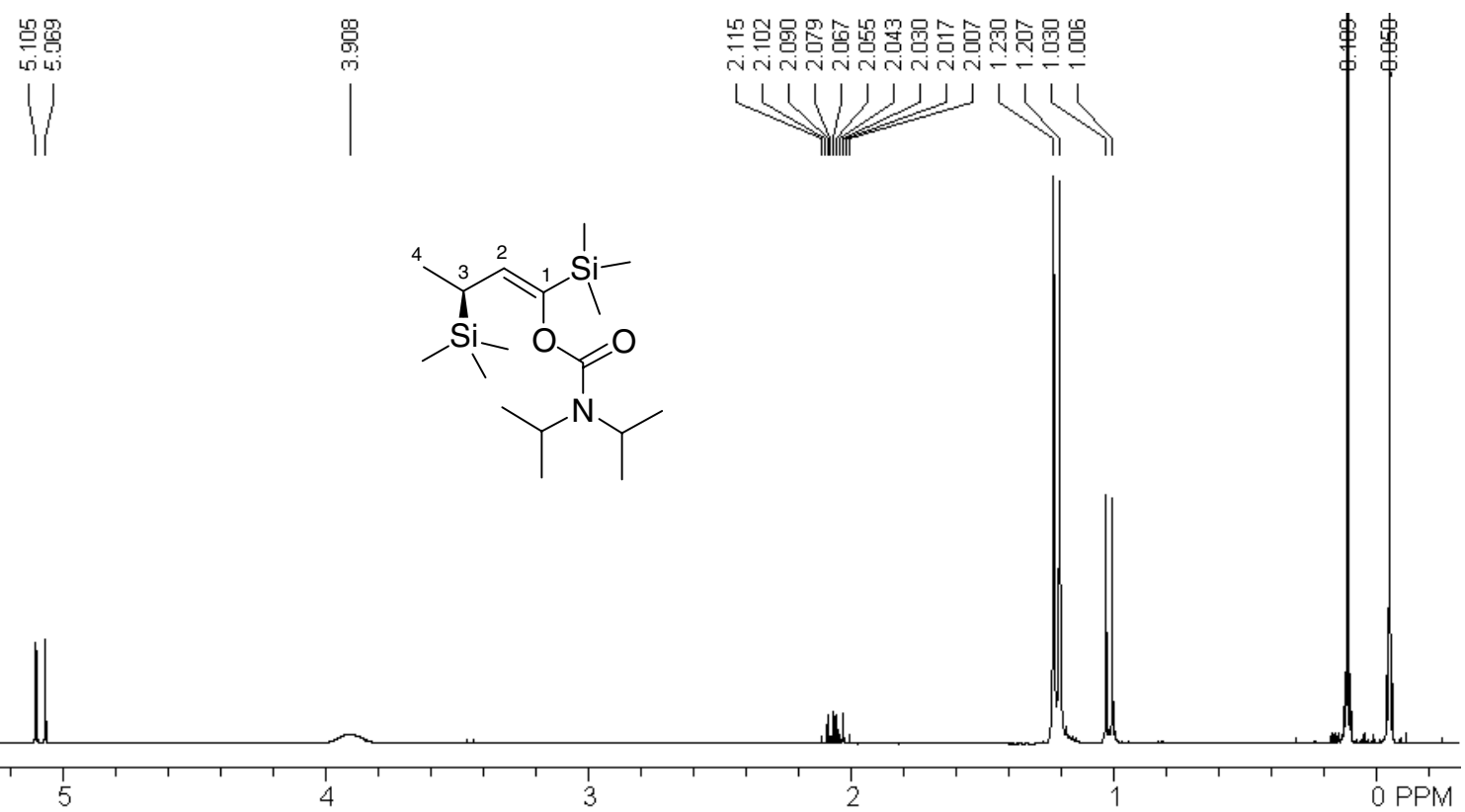

5.2 (-)-(1E,3S,4R)-4-Hydroxy-3,5-dimethyl-1-trimethylsilyl-1-hexenyl $N, N$ Diisopropylcarbamate (anti-6b)
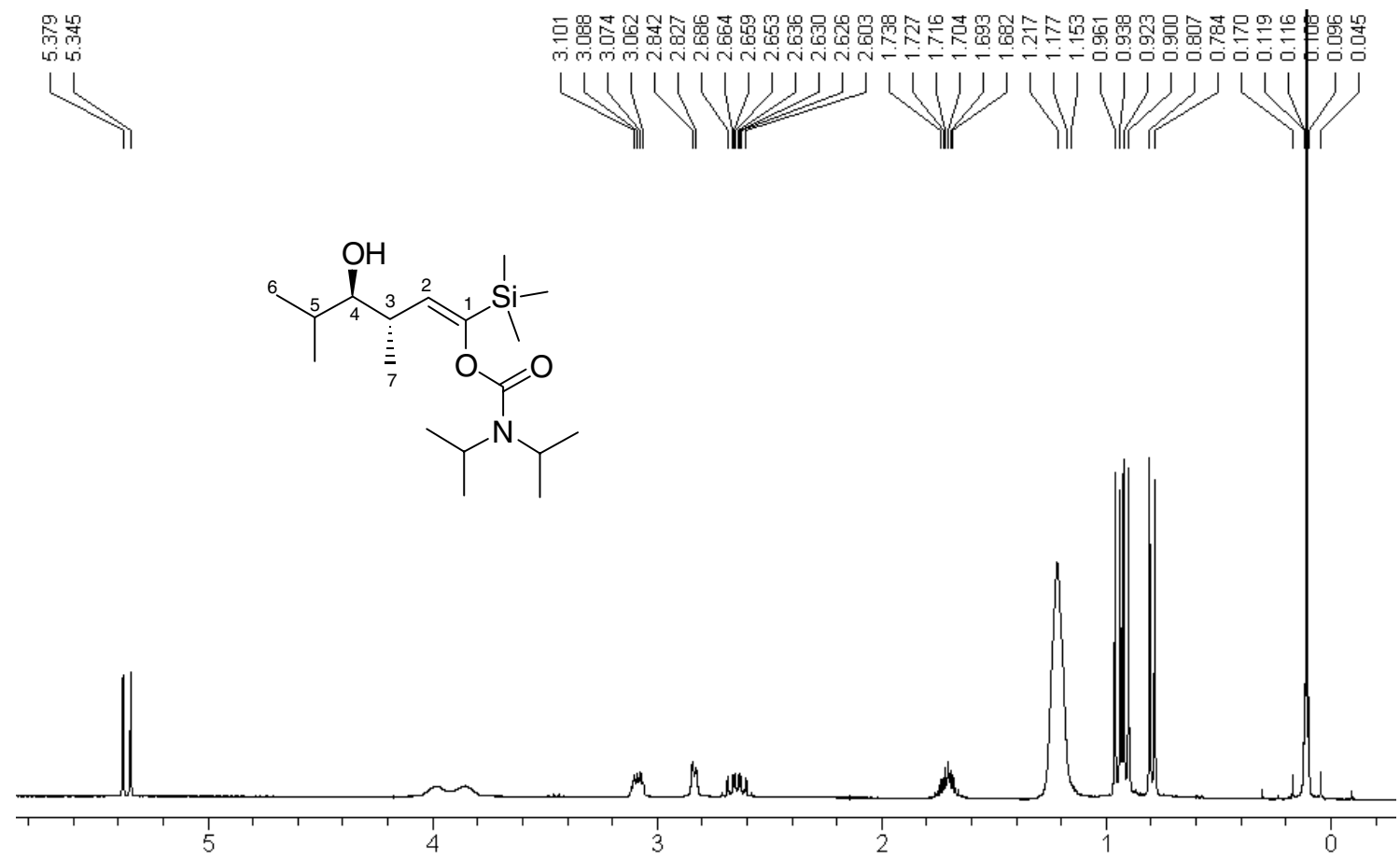
5.3 (+)-(1E,3S)-3-Methoxycarbonyl-3-phenyl-1-trimethylsilyl-1-propenyl $N, N$ Diisopropylcarbamate (ent-9d)

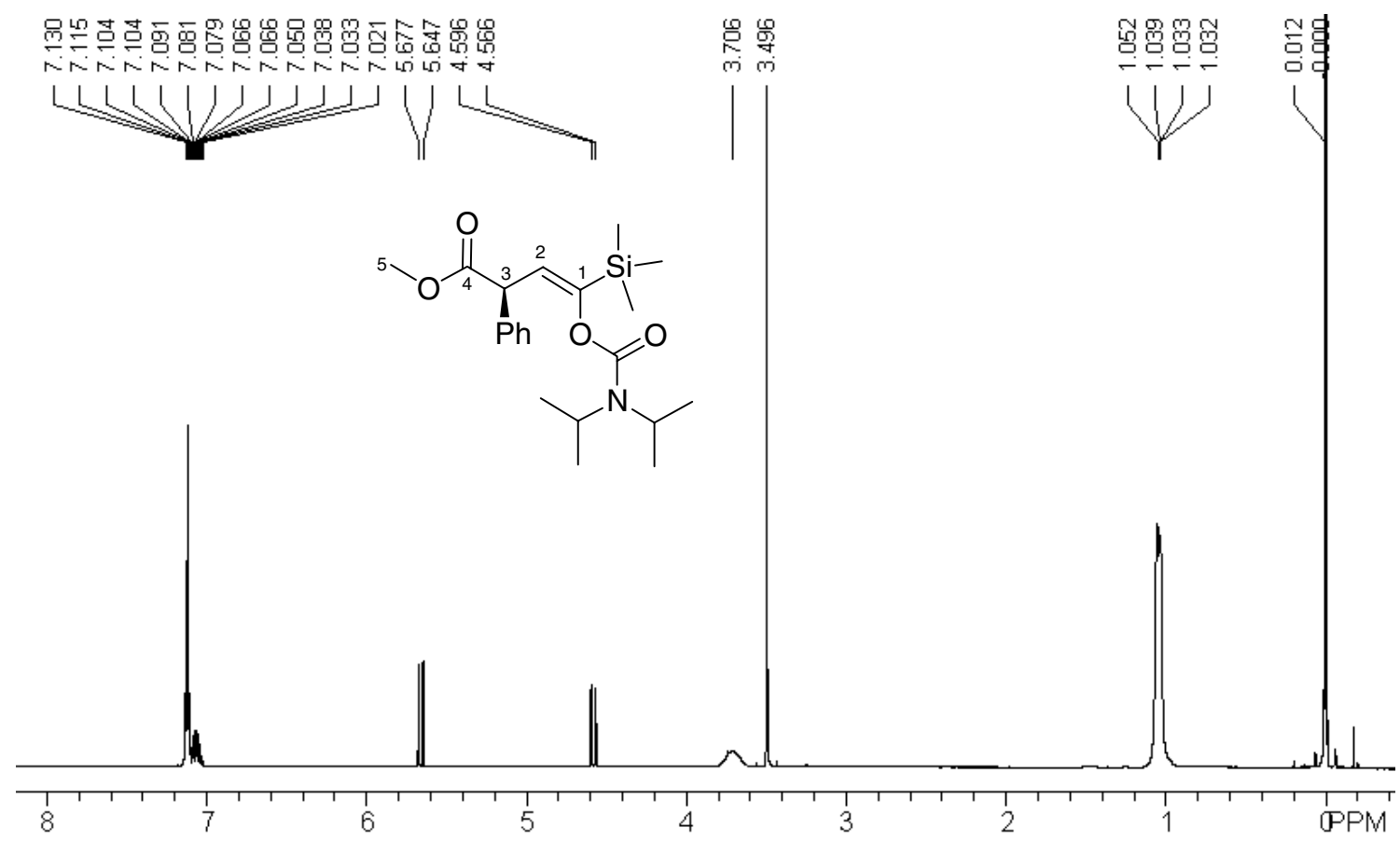

5.4 (-)-(1E,3R,4R)-4-Hydroxy-5-methyl-3-phenyl-1-trimethylsilyl-1-hexenyl $N, N$ Diisopropylcarbamate (anti-11b)
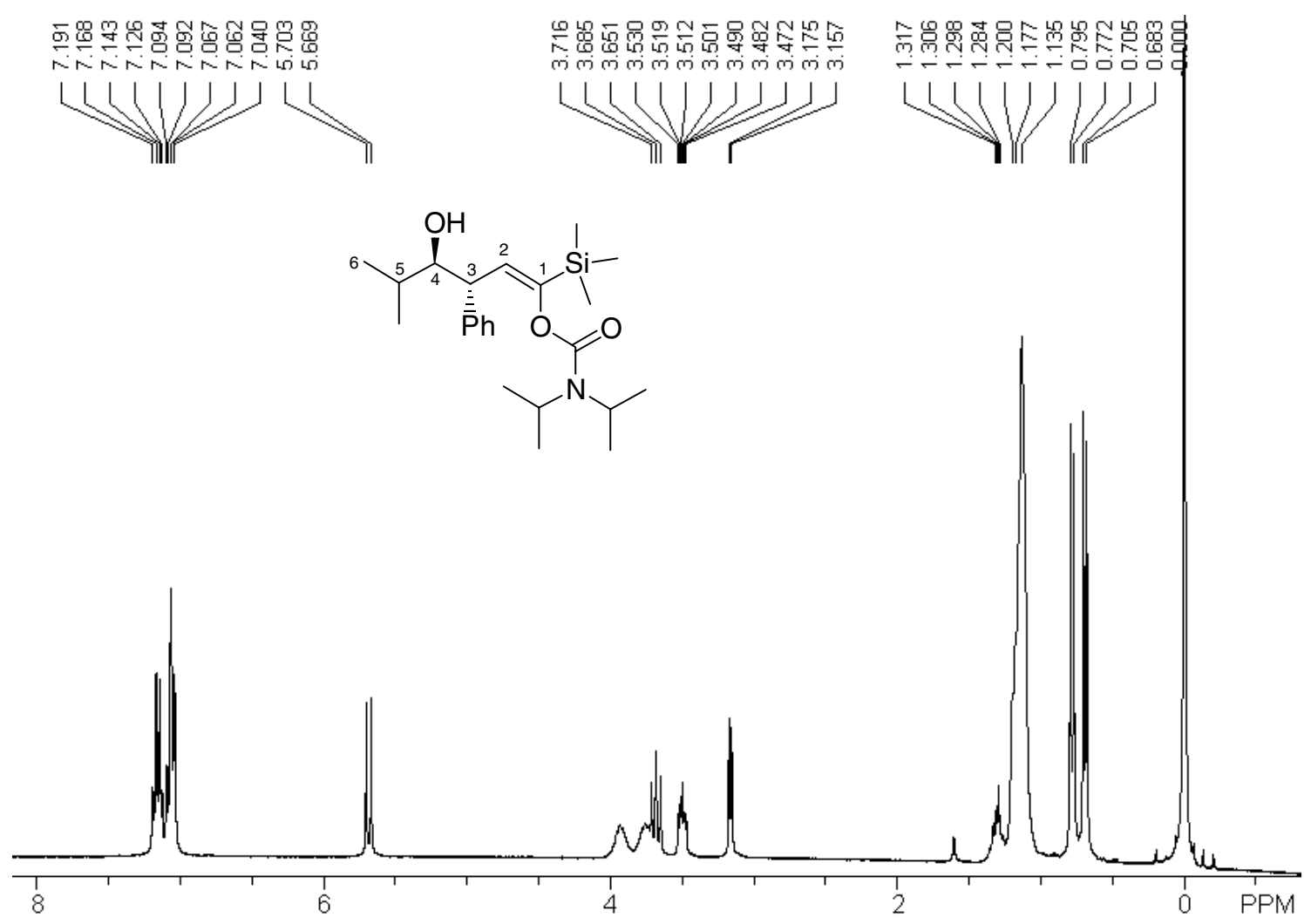
